\title{
NOCIONES DE REACCIÓN QUÍMICA EN EDUCACIÓN INICIAL MEDIANTE ACTIVIDAD EXPERIMENTAL
}

\section{CHEMICAL REACTION NOTION IN INITIAL EDUCATION USING LABORATORY ACTIVITY}

\author{
Wilmer López¹, José Escalona², Yelitza Guillén ${ }^{3}$, Yuly Lema4 ${ }^{4}$ Mayra Ponce $^{5}$
}

\begin{abstract}
${ }^{1}$ Magister Química Analítica, profesor agregado Facultad de Humanidades y Educación, Universidad de los Andes, Mérida, Venezuela, Igwilmer@yahoo.com; ${ }^{2}$ Magister Química Orgánica, profesor agregado Facultad de Humanidades y Educación, Universidad de los Andes, Mérida, Venezuela, cieduc@ula.ve; ${ }^{3}$ Licenciado Educación Preescolar, docente Ministerio de Educación, yelitza_82_5@hotmail.com; ${ }^{4}$ Licenciado Educación Preescolar, docente Ministerio de Educación, ylema@ hotmail.com; ${ }^{5}$ Licenciado Educación Preescolar, docente Ministerio de Educación, mayraponce_21@yahoo.com.
\end{abstract}

Rev. U.D.C.A Act. \& Div. Cient. 13 (1): 157-162, 2010

\section{RESUMEN}

Abordar la investigación sobre la influencia de los métodos activos en el aprendizaje, se justifica por su impacto en la transformación didáctica. La presente investigación tuvo como objetivo estudiar la formación de nociones químicas en niños de cinco a seis años, usando la experimentación. Metodológicamente, se empleó un paradigma crítico e interpretativo, por tanto, el estudio fue de estilo cualitativo y realizado con diez menores participantes. Los resultados muestran que la actitud del grupo de niños cambia durante la actividad empírica y son capaces de describir algunos fenómenos propios de las reacciones químicas. Finalmente, se concluyó que la experimentación facilita la construcción de la noción de reacción química y favorece el proceso de aprendizaje.

Palabras clave: Nociones, didáctica de la química, estrategias, reacción química, aprendizaje.

\section{SUMMARY}

Focusing the research on the influence of the active methods in learning is justified due to their impact on teaching transformation. The objective of the present study was to assess the formation of chemical notions in children five and six years old, through the use of experimentation. Methodologically, a critical and interpretative paradigm was applied; therefore, the research had a qualitative style and was conducted with ten participants. Results show that in the group, the children's attitudes change during the taskexperimentation, and that they are capable of describing some phenomena which are characteristic of chemical reactions. Finally, it was concluded that experimentation not only facilitates the construction of the notion of chemical reaction but also enhances the learning process.

Key words: Notions, didactics of the chemistry, strategies, chemical reaction, learning.

\section{INTRODUCCIÓN}

El aprendizaje de las ciencias naturales supone un gran desafío, tanto para el docente como para el alumno, puesto que las distintas áreas que la conforman son impartidas de forma abstracta, parcelada y ajena a las situaciones que se viven cotidianamente, es decir, sin establecer relaciones con ciertos fenómenos propios del ambiente, en el cual, se desenvuelven los niños y niñas.

De esta manera, procesos como el aprendizaje significativo, por ejemplo, se ve coartado por este 
proceder. Novak (1988) afirma que un aprendizaje significativo posibilita la adquisición de grandes cuerpos integrados de conocimiento y para ello, es necesario que el aprendiz transforme y estructure los datos; este proceso permite que la información exterior se interrelacione e interactúe con los esquemas de conocimientos previos, por tanto, el aprendizaje implica una reestructuración activa de las percepciones. A partir de lo planteado, la enseñanza de las ciencias, se debe concebir como un proceso de aprendizaje significativo, en el que se tomen en cuenta las ideas previas de los estudiantes (Harlen, 1999; Osborne \& Freyberg, 1998; Trinidad E Garritz, 2003; Bello, 2004) y considerar que la discusión de conceptos tiene como característica formar nuevas relaciones de significados (Bruner, 2001). Estas relaciones organizadas son las que permiten ampliar la noción científica de los fenómenos cotidianos de las ciencias.

El presente estudio, da como resultado que niñas y niños forman ideas conceptuales referidas a la noción de reacción química, a partir de actividades experimentales, en las cuales, tienen la oportunidad para que manifiesten oralmente sus ideas respecto a cada experiencia y fenómenos observados, como manifestación del mundo micro (Gómez, 1996). Es necesario destacar que tomando en cuenta el nivel de abstracción de la muestra en estudio es que se ha planteado la construcción de la noción de reacción química, de manera que niñas y niños puedan ir progresivamente modificando, tanto su estructura cognitiva como los nuevos elementos que la transforman, mediante los procesos de acomodación y asimilación (Sosa, 2004). Sin embargo, es en la reflexión activa, aunada a una intensa operación mental entre los conocimientos previos y los nuevos que el individuo va construyendo sus conceptos científicos, realizando un esfuerzo intelectual para darle coherencia y sentido a sus nuevas ideas (Campanario \& Otero, 2000), fundamental en el tan ansiado cambio conceptual (Garritz, 2001; Carretero, 1997; Pozo, 2007) y que se espera logren los estudiantes de posteriores niveles de escolaridad. Y este cambio conceptual, se espera sea alcanzado durante la práctica educativa, con una adecuada supervisión y actuación docente, en situaciones que implican la toma de decisiones didáctico-éticas, que tienen que ver con la forma de abordar los problemas para hacerlos más reales, cercanos y con un alto grado de responsabilidad del estudiante (Martínez \& Fandiño, 2009). Por ello, pensamos que el tema de la transformación didáctica para un mejor aprendizaje pasa por el hecho de asumir una forma diferente de pensar el proceso educativo, en otras palabras, un modelo diferente de los valores éticos en la didáctica.

Por esta razón, planteamos que abordar la formación de nociones científicas desde temprana edad debería conducir a la disminución de la tendencia de tener futuros estudiantes con una cantidad considerable de ideas erróneas sobre acontecimientos naturales y cotidianos. Esto último, nos habla claramente de un proceso ético-didáctico, donde la actividad científica no solamente se piensa para niñas y niños, sino que además se concibe para que sean ellos quienes la desarrollen. En este sentido, en el Currículo de Educación Inicial del Ministerio de Educación, Cultura y Deporte (2005), se señala que en la educación preescolar la enseñanza de las ciencias está dirigida a que los niños y niñas identifiquen los elementos de su entorno, explicándose progresivamente los acontecimientos sociales y naturales, a través de la observación, la experimentación, la formulación y la comprobación de hipótesis, de manera que desplieguen capacidades efectivas y valorativas, como ser integrante del ambiente. Y este es un objetivo prioritario en la actividad y en el enfoque didáctico de los maestros.

\section{MATERIALES Y MÉTODOS}

El objetivo de este trabajo fue estudiar el proceso de aparición de las nociones de reacción química, mediante la experimentación con niñas y niñas, de cinco a seis años de edad.

El carácter de la investigación fue de tipo exploratoriodescriptivo y con enfoque de naturaleza cualitativa. En este marco, los datos pueden combinar fracciones cuanti-cualitativas para hacer una descripción exhaustiva del contexto en estudio (Bisquera, 1989). Se hace notar que la naturaleza del problema constituye un condicionante de la metodología (Arnal et al. 1992). Se considera al proceso de investigación descriptiva como un sistema interpretativo donde diversos elementos actitudinales, conceptuales y procesuales determinan un contexto histórico (Best, 1981). Así pues, queda en evidencia que se optó por este enfoque, dada la posibilidad que ofrece para presentar sistematizaciones diversas sobre la situación en estudio (Feliz \& Ricoy, 2003). Además, la investigación cualitativa es un proceso que involucra varios elementos, como exploración de 
aspectos sociales, explicación de interrogantes que surgen en la situación de estudio y aplicación de los resultados para mejorar los conocimientos sobre nuestro problema (Huber, 2003). De este modo, el método queda plenamente referenciado hacia el contexto de las respuestas suministradas por los participantes y sus posiciones frente a la temática estudiada.

El diseño de campo, se basó en la experimentación y en el patrón del antes, durante y después del desarrollo de las actividades experimentales. Bajo esta concepción, la investigación es propicia para reconocer cómo se adelanta el proceso de aprendizaje de las nociones químicas y de qué forma la muestra se manifiesta, puesto que existe la posibilidad de mostrar el por qué de algunos eventos cotidianos, a través de experiencias, en las que los procesos, tales como la observación, la formulación de hipótesis, entre otros, pueden incentivar a niñas y niños al estudio y disfrute por la química.

Para realizar este estudio, se seleccionó una muestra de diez menores, en edades comprendidas entre cinco y seis años, del nivel de educación inicial, pertenecientes a una población de cien estudiantes de una institución pública del Estado Mérida - Venezuela. A fin de sistematizar la información, se realizaron seis actividades experimentales (Vancleave, 2001), una por día; niñas y niños fueron codificados con números del uno al diez; así mismo, se grabaron las opiniones que ellos aportaron antes, durante y finalizada cada actividad experimental, para su posterior análisis y discusión.

\section{DESCRIPCIÓN DE LAS EXPERIENCIAS}

Experiencia 1. La bebida espumosa: Para realizar esta actividad, se llenó un frasco pequeño a la mitad con bebida carbonatada, se le agregó una cucharadita de sal, formando burbujas y aparentando espuma sobre la superficie de la bebida; cada burbuja se llena de dióxido de carbono, dado que la sal (sólida) desplazó al gas dióxido de carbono; a este proceso se le llama efervescencia. En este caso, cabe recordar que existe una reacción química de descomposición donde:

$$
\mathrm{H}_{2} \mathrm{CO}_{3}(\mathrm{ac}) \rightarrow \mathrm{CO}_{2}(\mathrm{~g}) \uparrow+\mathrm{H}_{2} \mathrm{O}(\mathrm{l})
$$

En esta reacción, se puede notar el desprendimiento de dióxido de carbono, y, por tanto, es posible su posterior desplazamiento por el sólido.
Experiencia 2. El huevo desnudo: Se colocó un huevo crudo en el frasco de vidrio; luego, se agregó vinagre hasta cubrirlo totalmente y se observó durante las 24 horas siguientes. Inmediatamente, comenzó a formarse burbujas sobre la superficie del cascaron y su número aumentó al pasar el tiempo. Después de un día, el cascarón desapareció y partes de él quedaron flotando en la superficie del vinagre, mientras que el huevo permaneció intacto, porque está recubierto con una membrana transparente. Lo observado, se debe a que existe una reacción química entre el ácido acético (vinagre) y el carbonato de calcio (compuesto de la concha del huevo) con desprendimiento de dióxido de carbono, representado de la manera siguiente:

$$
\begin{gathered}
\mathrm{CaCO}_{3}(\mathrm{~s})+2 \mathrm{CH}_{3} \mathrm{COOH}(\mathrm{ac}) \rightarrow \mathrm{C}\left(\mathrm{CH}_{3} \mathrm{COO}\right)_{2}(\mathrm{ac})+ \\
\mathrm{CO}_{2}(\mathrm{~g}) \uparrow+\mathrm{H}_{2} \mathrm{O}(\mathrm{l})
\end{gathered}
$$

Experiencia 3. El color desaparece: Primeramente, se llenó un frasco a la mitad con agua y se agregaron dos gotas de colorante rojo para alimentos. Luego, se adicionó una gota de blanqueador a la mezcla anterior y, por último, se añadió una o dos gotas del mismo tinte. El agua de color rojo se decoloró a medida que el blanqueador traspasó. Posteriormente, se le volvió agregar una gota del colorante produciéndose un efecto interesante, donde éste desapareció, es decir, que la estructura química del colorante se ve afectada por el poder oxidante del blanqueador, a través de una reacción química.

Experiencia 4. El corcho saltarín: Se colocó vinagre en una botella, agregándole bicarbonato y cubriéndolo con un tapón de corcho, el cual, no se ajustó demasiado. Al producirse dióxido de carbono dentro de la botella, la presión se incrementó y el corcho saltó con bastante fuerza. Cabe destacar que la producción de dióxido de carbono se explica cuando reacciona el bicarbonato con el vinagre de la manera siguiente:

$$
\begin{gathered}
\mathrm{NaHCO}_{3}(\mathrm{~s})+\underset{\mathrm{CO}_{2}(\mathrm{~g}) \uparrow+\mathrm{H}_{2} \mathrm{O}(\mathrm{l})}{\mathrm{CH}_{3} \mathrm{COOH}(\mathrm{ac}) \rightarrow \mathrm{CH}_{3} \mathrm{COONa}(\mathrm{ac})+}
\end{gathered}
$$

Experiencia 5. La escritura mágica: Esta actividad consistió en recortar un pedazo de papel donde se escribió un mensaje, empleando un pincel mojado con jugo de limón. Luego, se dejó secar y, finalmente, se sumergió en una solución de tintura de iodo. Como resultado, se formó color azul (almidón-iodo) por fuera 
de la escritura y ésta permaneció incolora (ácido del limón-iodo). En esta reacción, la celulosa del papel se une con el iodo formando un complejo de color azul y en la zona ácida, no.

Experiencia 6. Volcán en erupción: Para desarrollar la última actividad, se mezcló polvo para hornear y vinagre teñido con colorante (rojo) para alimentos en una botella de bebida, que se cubrió con tierra húmeda en forma de volcán. Se observó efervescencia química por el dióxido de carbono (reacción química de la experiencia 4).

\section{RESULTADOS Y DISCUSIÓN}

Experiencia 1. En este primer experimento, los diez menores, al comenzar la actividad, expresaron que al abrir la bebida gaseosa se produjo un sonido, que denominaron "gas". Esta expresión demuestra que han estado en contacto con ciertas experiencias en su vida cotidiana, para llegar hacer tal afirmación. También se observó que la mayoría de respuestas se limitaron a describir la situación sin ir más allá de lo que sucedía. En este sentido, Harlen (1999) señala lo siguiente: "los niños de cinco a siete años se centran en un sólo aspecto del objeto o situación y su idea de causa efecto se funda en la presencia de algunas características u objetos sin extenderse a un mecanismo".

En esta actividad los niños y niñas no fueron más allá de lo observado concretamente durante la experiencia; también se evidenció la tendencia de la muestra en hacer asociaciones que iban de lo particular a lo particular. Por ejemplo, tres menores manifestaron, al observar la bebida gaseosa, que las "burbujas, suben y bajan, y hay chispitas que salen por arriba", esto es lo que Piaget denominó "Procesos Transductivos" (Shaffer, 2000; Wiener E Dulcan, 2006). Ocho de los diez niños y niñas expresaban que "al refresco le salía espuma, porque se le colocó sal" y, en cierto modo, esto era lo que sucedía a simple vista; no obstante, hasta ese momento, los estudiantes no contaban con los elementos necesarios para dar una explicación con mayor profundidad al fenómeno observado.

Experiencia 2. Durante esta experiencia, diez niñas y niños realizaron comparaciones coherentes entre dos situaciones, el antes y después del ensayo e hicieron uso del término flotación, además, la mayoría de las afirmaciones (ocho niños y niñas), se basaron en que había ocurrido un cambio, hecho que se demuestra en respuestas como: "está grande y la concha se le salió por el vinagre", "se ve grande y está desnudo", "la concha se va disolviendo por el vinagre y parece una pelota grande de goma", entre otras. De esta manera, al introducir la noción de cambio en las respuestas, se produce un importante paso hacia lo que significa reacción química.

Experiencia 3. Ante este experimento, diez menores respondieron que la desaparición del color se debía a la colocación de un blanqueador a la solución. Por ejemplo: "está cambiando de color como la ropa", "cambió de color porque le echamos mucho cloro"; en estas afirmaciones, se pone en evidencia la idea de causa y efecto, que es peculiar a esta edad, que se funda en la presencia de alguna característica u objeto sin extenderse a un mecanismo (Harlen, 1999), es decir, las respuestas estuvieron acordes a la edad y desarrollo cognitivo.

Además, respuestas como "el blanqueador se comió el color", se encuentran dotadas de uno de los elementos propios del pensamiento infantil, como lo es el "animismo", que consiste en la tendencia de atribuir a los objetos y a los hechos físicos las características de las entidades biológico-psicológica, es decir, a dotarlos de vida, de consciencia, de voluntad, entre otros (UNA - UPEL, 1993). Pero aún cuando sus afirmaciones son animistas en el fondo tienen presente que el cloro debe contener una sustancia que actúa sobre el color haciendo que éste desaparezca.

Experiencia 4. En el desarrollo de esta experiencia se les preguntó a los niños y niñas ċqué sucederá si se une el vinagre y el bicarbonato? En sus respuestas, cuatro predijeron que "el corcho se dispararía"; tres dijeron que "estallaría" al unirse estos dos elementos; un niño señaló que "estallaría porque tiene gases" y dos de ellos señalaron que el corcho "volaría", es decir, tienen la noción sobre la producción de gases, lo que hace que el corcho se dispare con fuerza.

En esta experiencia hay una reacción química donde se produce $\mathrm{CO}_{2}$, que comienza a ser descrita por niñas y niños, al expresar lo que sucedía: uno de ellos manifestó que era producto de los "gases"; cinco niños y niñas "por las burbujas que habían en la botella" y cuatro menores "porque se unió el bicarbonato con el vinagre 
y al agitarlo se disparó el corcho”. Todo ello evidencia un incremento sustancial de predicción al ir agregando elementos significativos, que van complementando la construcción de su estructura conceptual, sobre la noción de reacción química.

Experiencia 5. Se puede inferir que niñas y niños atribuían a la zona impregnada con limón una característica de transparencia, afirmando, ocho de ellos, que "no se escribió nada" y dos niños y niñas "escribimos pero no se ve nada, porque el color del jugo es claro". Luego, se les preguntó qué sucedería si la hoja se sumerge en agua con iodo; la mayoría de ellos afirmaron que se mancharía o se pintaría. Así mismo, se indagó también por qué sumergida la hoja en iodo se pudo observar las grafías que se hicieron con el jugo de limón y las respuestas estuvieron basadas en el uso de limón y colorante, tales como: "se ven las letras", "se ven puras manchas", "se ve manchado", "las letras no se pintaron porque tenían jugo de limón", es decir, asignándoles características específicas a éste. Esto indica que los menores saben que el espacio cubierto por limón no se colorea con el iodo aunque no puedan dar una explicación más compleja, debido al nivel de su desarrollo cognitivo.

Experiencia 6. En esta actividad experimental antes de mezclarse los ingredientes, niños y niñas hicieron algunas afirmaciones, tales como: "saldrá espuma", "estallará", "al unirse el vinagre con el polvo saldrá espuma", "pasará lo mismo que con el corcho saltarín", entre otras. Al igual que la experiencia cuatro, la reacción del bicarbonato con el vinagre produce dióxido de carbono, el cual, es un gas que se desprende produciendo el burbujeo o espuma, como fue descrito por los menores. En consecuencia, se puede decir que la muestra hizo una predicción en cuanto a que se iba a producir un cambio en los materiales, todo ello con base a los elementos conceptuales internalizados, a partir de las experiencias previas.

Basado en el análisis de las experiencias anteriores, se puede afirmar que en la medida que se ejecutaron las actividades experimentales niñas y niños fueron incorporando, progresivamente, nuevos elementos lingüísticos que suponen la aparición de noción de reacción química; tal es el caso del experimento tres y cuatro, en donde los niños manifestaron que la unión del bicarbonato y el vinagre produce cambios químicos.
Otra tendencia importante que se evidenció fue que niñas y niños, haciendo uso de sus propios elementos de lenguaje y en el marco de su nivel de abstracción científica, trataron de dar explicación a algunos hechos que observaron en las actividades desarrolladas, como es el caso del experimento número dos, en el que un niño manifestó que "la concha se le va saliendo porque se le echó vinagre". En el experimento seis algunos participantes expresaron "porque se unieron todos los ingredientes”, lo que evidencia que la muestra procuró una explicación a los fenómenos observados, de acuerdo a su capacidad cognitiva y nivel de abstracción.

También se advirtió que niñas y niños realizaron comparaciones en la medida que transcurría y se desarrollaba una nueva experiencia demostrativa, como fue el caso de la práctica seis, en la que dos niños manifestaron: "Lo mismo pasó con el corcho saltarín cuando le echamos el bicarbonato al vinagre", lo cual, hace evidente que realizan una generalización sobre los fenómenos, algo muy típico del razonamiento inductivo en las ciencias.

Igualmente, se descubrió que la capacidad de predicción se incrementó a partir de los experimentos realizados, destacando que la muestra distinguió las propiedades de los materiales antes y después de mezclados, cuando pronosticaron la posibilidad de producción de burbujas o de una explosión (Experiencia 4), es decir, consideraron un cambio en los materiales, característica intrínseca en una reacción química.

Así, queda en evidencia que a partir de la puesta en práctica de actividades experimentales se ayuda considerablemente a abordar, de una manera sencilla y divertida, temas relacionados con química, lo cual es importante para formar una estructura conceptual básica, que se irá transformando y enriqueciendo en la medida que niñas y niños van incorporando nuevos elementos en sus siguiente años escolares.

Conflicto de intereses: Hacemos constar que el manuscrito fue revisado y preparado por los autores quienes declaramos que no existe ningún conflicto de intereses que ponga en riesgo los resultados presentados. 


\section{BIBLIOGRAFÍA}

1. ARNAL, J.; DEL RINCÓN, D.; LATORRE, A. 1992. Investigación Educativa. España: Editorial Labor. 219p.

2. BELLO, S. 2004. Ideas Previas y Cambio Conceptual. Educación Química. 15(3):210-217.

3. BEST, J. 1981. Cómo investigar en educación. España: Editorial Morata. 178p.

4. BISQUERA, R. 1989. Métodos de una investigación educativa. España: Ed. CEAC. 312p.

5. BRUNER, J. 2001. El Proceso Mental en el Aprendizaje. España: Editorial Narcea, S.A. 425p.

6. CAMPANARIO, J.; OTERO J. 2000. Más allá de las Ideas Previas como Dificultades de Aprendizaje; Las pautas del Pensamiento, las Concepciones Epistemológicas y las Estrategias Metacognitivas. Enseñanza de las Ciencia. 18(2):155-169.

7. CARRETERO, M. 1997. Construir y Enseñar las Ciencias Experimentales. $2^{\text {da }}$ Edición. Argentina: Editorial, Aique, 387p.

8. FELIZ, T.; RICOY, M. 2003. El descubrimiento de la dimensión cualitativa de la investigación a través de un foro educativo. En: Medina, A.; Castillo, S. (Coord). Metodología para la realización de proyectos de investigación y tesis doctorales. España: Ed. Universitas, p.131-165.

9. GARRITZ, A. 2001. Veinte años de la teoría del cambio conceptual. Educación Química. 12(3):123-126.

10. GÓMEZ, M. 1996. Ideas y dificultades en el aprendizaje de la química. Alambique. 3(7):37-44.

11. HARLEN, W. 1999. Enseñanza y Aprendizaje de las Ciencias. España, Editorial Morata S.L. 415p.

12. HUBER, G. 2003. Introducción al análisis de datos cualitativos. En: Medina, A.; Castillo, S. (Coord). Metodología para la realización de proyectos de investigación y tesis doctorales. España: Editorial Universitas, p.91-129.
13. MARTÍNEZ, L.; FANDIÑO, V. 2009. Implicaciones éticas que enfrenta el docente al supervisar la práctica clínica de estudiantes de enfermería Rev. U.D.C.A. Act. \& Div. Cient. 12(1):33-41.

14. MINISTERIO DE EDUCACIÓN CULTURA Y DEPORTE. 2005. Currículo de Educación Inicial. Venezuela: Imprenta Nacional. 523p.

15. NOVAK, J. 1988. Constructivismo Humano: un consenso emergente. Enseñanza de la Ciencias. 6(3): 213-223.

16. OSBORNE, R.; FREYBERG, P. 1998. El Aprendizaje de las Ciencias. Implicaciones de la "Ideas Previas"

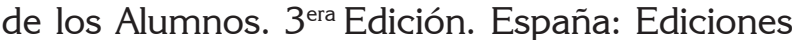
NARCEA, S.A. 301p.

17. POZO, J.I. 2007. Cambio conceptual y representacional en el aprendizaje y la enseñanza de la ciencia. Volumen CLII colección Aprendizaje. Madrid: A. Machado Libros, S.A. 311p.

18. SHAFFER, D. 2000. Psicología del desarrollo: infancia y adolescencia. México: Internacional Thomson Editores. 641p.

19. SOSA, P. 2004. Química Aritmética. Un primer paso hacia el cambio conceptual. Educación Química. 15(3):248-255.

20. TRINIDAD, R.; GARRITZ, A. 2003. Revisión de las concepciones alternativas de los estudiantes de Secundaría sobre la estructura de la materia. Educación Química 14(2):72-85.

21. UNA - UPEL. 1993. Psicología del Desarrollo. Volumen 1. El Desarrollo Cognoscitivo del Niño en Edad Preescolar. Venezuela: Editorial UNA. 192p.

22. VANCLEAVE, J. 2001. Química para Niños y Jóvenes. México: Ed. Limusa-Noriega, 201p.

23. WIENER, J.; DULCAN, M. 2006. Tratado de Psiquiatría de la Infancia y la Adolescencia. España: Ed. Masson. 397p.

Recibido: Octubre 12 de 2009

Aceptado: Mayo 4 de 2010 